\title{
Intuitionistic Fuzzy Implications and the Axioms of Intuitionistic Logic
}

\author{
Nora A. Angelova ${ }^{1}$ Krassimir T. Atanassov $^{1}$ \\ ${ }^{1}$ Department of Bioinformatics and Mathematical Modelling \\ Institute of Biophysics and Biomedical Engineering \\ Bulgarian Academy of Sciences \\ Acad. G. Bonchev Str., Bl. 105, Sofia-1113, Bulgaria \\ e-mails: nora.angelova@biomed.bas.bg and krat@bas.bg
}

\begin{abstract}
During years of research, there have been defined 153 intuitionistic fuzzy implications. In the paper, it is checked which of these implications satisfy which axioms of the intuitionistic logic, whether as (classical) tautologies or as intuitionistic fuzzy tautologies. Some open problems, related to the operations of intuitionistic fuzzy propositional calculus, are formulated.
\end{abstract}

Keywords: Implication, Intuitionistic fuzzy logic, Intuitionistic logic.

\section{Introduction}

In a series of publications, (see, e.g. $[1,2]$ ), 153 different operations "implication" in intuitionistic fuzzy propositional calculus, have been introduced. They have been found to generate 45 operations "negation" over intuitionistic fuzzy sets.

In the present paper, we study which of these implications satisfy which axioms of the intuitionistic logic, and whether, doing so, they behave tautologies or as intuitionistic fuzzy tautologies.

In intuitionistic fuzzy propositional calculus, if $x$ is a variable, then its truth-value is represented by the ordered couple

$$
V(x)=\langle a, b\rangle
$$

so that $a, b, a+b \in[0,1]$, where $a$ and $b$ are degrees of validity and of non-validity of $x$.

For the needs of the discussion below, we shall define the notion of Intuitionistic Fuzzy Tautology (IFT, see, e.g. [1, 2]) by:

\section{$x$ is an IFT if and only if}

for $V(x)=\langle a, b\rangle$ it holds that $a \geq b$,

while

$$
\begin{gathered}
x \text { will be a (classical) tautology if and only if } \\
\qquad a=1 \text { and } b=0 .
\end{gathered}
$$

As in the case of ordinary logics, $x$ is a tautology, if $V(x)=\langle 1,0\rangle$. Obviously, each tautology is intuitionistic fuzzy tautology, but the opposite is not true.

\section{On the operations "implication" and "negation" in intuitionistic fuzzy propositional calculus}

Below, we shall assume that for the two variables $x$ and $y$ the equalities: $V(x)=\langle a, b\rangle, V(y)=\langle c, d\rangle$ $(a, b, c, d, a+b, c+d \in[0,1])$ hold.

First, for the two variables $x$ and $y$, we define the operations "conjunction" (\&) and "disjunction" ( $\vee$ (see, e.g. $[1,2])$ by:

$$
\begin{aligned}
& V(x \& y)=\langle\min (a, c), \max (b, d)\rangle, \\
& V(x \vee y)=\langle\max (a, c), \min (b, d)\rangle .
\end{aligned}
$$

In some definitions, we need to use the auxiliary functions sg and $\overline{\mathrm{sg}}$ defined by,

$$
\begin{aligned}
& \operatorname{sg}(x)=\left\{\begin{array}{ll}
1 & \text { if } x>0 \\
0 & \text { if } x \leq 0
\end{array},\right. \\
& \overline{\operatorname{sg}}(x)= \begin{cases}0 & \text { if } x>0 \\
1 & \text { if } x \leq 0\end{cases}
\end{aligned}
$$

The list of all currently existing intuitionistic fuzzy implications and negations are given in Tables 1 and 2, respectively. Another JavaScript powered visualization of these operations with dynamically sortable membership and non-membership parts is given online in $[7,8]$.

Table 1

\begin{tabular}{|l|l|}
\hline$\rightarrow_{1}$ & $\langle\max (b, \min (a, c)), \min (a, d)\rangle$ \\
\hline$\rightarrow_{2}$ & $\langle\overline{\operatorname{sg}}(a-c), d \cdot \operatorname{sg}(a-c)\rangle$ \\
\hline$\rightarrow_{3}$ & $\langle 1-(1-c) \cdot \operatorname{sg}(a-c)), d \cdot \operatorname{sg}(a-c)\rangle$ \\
\hline$\rightarrow_{4}$ & $\langle\max (b, c), \min (a, d)\rangle$ \\
\hline$\rightarrow_{5}$ & $\langle\min (1, b+c), \max (0, a+d-1)\rangle$ \\
\hline$\rightarrow_{6}$ & $\langle b+a c, a d\rangle$ \\
\hline$\rightarrow_{7}$ & $\langle\min (\max (b, c), \max (a, b), \max (c, d))$, \\
& $\max (\min (a, d), \min (a, b), \min (c, d))\rangle$ \\
\hline$\rightarrow_{8}$ & $\langle 1-(1-\min (b, c)) \cdot \operatorname{sg}(a-c)$, \\
& $\max (a, d) \cdot \operatorname{sg}(a-c), \operatorname{sg}(d-b)\rangle$ \\
\hline$\rightarrow_{9}$ & $\left\langle b+a^{2} c, a b+a^{2} d\right\rangle$ \\
\hline$\rightarrow_{10}$ & $\langle c \cdot \overline{\operatorname{sg}}(1-a)+\operatorname{sg}(1-a) \cdot(\overline{\operatorname{sg}}(1-c)$ \\
& $+b \cdot \operatorname{sg}(1-c)), d \cdot \overline{\operatorname{sg}}(1-a)$ \\
& $+a \cdot \operatorname{sg}(1-a) \cdot \operatorname{sg}(1-c)\rangle$ \\
\hline
\end{tabular}




\begin{tabular}{|c|c|}
\hline$\rightarrow_{11}$ & $\langle 1-(1-c) \cdot \operatorname{sg}(a-c), d \cdot \operatorname{sg}(a-c) \cdot \operatorname{sg}(d-b)\rangle$ \\
\hline$\rightarrow_{12}$ & $\langle\max (b, c), 1-\max (b, c)\rangle$ \\
\hline$\rightarrow_{13}$ & $\langle b+c-b . c, a . d\rangle$ \\
\hline$\rightarrow_{14}$ & $\begin{array}{l}\langle 1-(1-c) \cdot \operatorname{sg}(a-c)-d \cdot \overline{\operatorname{sg}}(a-c) \cdot \operatorname{sg}(d-b) \\
d \cdot \operatorname{sg}(d-b)\rangle\end{array}$ \\
\hline$\rightarrow_{15}$ & $\begin{array}{l}\langle 1-(1-\min (b, c)) \cdot \operatorname{sg}(a-c) \cdot \operatorname{sg}(d-b) \\
-\min (b, c) \cdot \operatorname{sg}(a-c) \cdot \operatorname{sg}(d-b), \\
1-(1-\max (a, d)) \cdot \operatorname{sg}(\overline{\operatorname{sg}}(a-c)+\overline{\operatorname{sg}}(d-b)) \\
-\max (a, d) \cdot \overline{\operatorname{sg}}(a-c) \cdot \overline{\operatorname{sg}}(d-b)\rangle\end{array}$ \\
\hline$\rightarrow_{16}$ & $\langle\max (\overline{\operatorname{sg}}(a), c), \min (\operatorname{sg}(a), d)\rangle$ \\
\hline$\rightarrow_{17}$ & $\left\langle\max (b, c), \min \left(a \cdot b+a^{2}, d\right)\right\rangle$ \\
\hline$\rightarrow_{18}$ & $\langle\max (b, c), \min (1-b, d)\rangle$ \\
\hline$\rightarrow_{19}$ & $\begin{array}{l}\langle\max (1-\operatorname{sg}(\operatorname{sg}(a)+\operatorname{sg}(1-b)), c) \\
\min (\operatorname{sg}(1-b), d)\rangle\end{array}$ \\
\hline$\rightarrow_{20}$ & $\langle\max (\overline{\operatorname{sg}}(a), \operatorname{sg}(c)), \min (\operatorname{sg}(a), \overline{\operatorname{sg}}(c))\rangle$ \\
\hline$\rightarrow_{21}$ & $\begin{array}{l}\langle\max (b, c \cdot(c+d)) \\
\left.\min \left(a \cdot(a+b), d .\left(c^{2}+d+c . d\right)\right)\right\rangle\end{array}$ \\
\hline$\rightarrow_{22}$ & $\langle\max (b, 1-d), 1-\max (b, 1-d)\rangle$ \\
\hline$\rightarrow_{23}$ & $\begin{array}{l}\langle 1-\min (\operatorname{sg}(1-b), \overline{\operatorname{sg}}(1-d)) \\
\min (\operatorname{sg}(1-b), \overline{\operatorname{sg}}(1-d))\rangle\end{array}$ \\
\hline$\rightarrow_{24}$ & $\langle\overline{\operatorname{sg}}(a-c) \cdot \overline{\operatorname{sg}}(d-b), \operatorname{sg}(a-c) \cdot \operatorname{sg}(d-b)\rangle$ \\
\hline$\rightarrow_{25}$ & $\begin{array}{l}\langle\max (b, \overline{\operatorname{sg}}(a) \cdot \overline{\operatorname{sg}}(1-b) \\
c \cdot \overline{\operatorname{sg}}(d) \cdot \overline{\operatorname{sg}}(1-c)), \min (a, d)\rangle\end{array}$ \\
\hline$\rightarrow_{26}$ & $\langle\max (\overline{\operatorname{sg}}(1-b), c), \min (\operatorname{sg}(a), d)\rangle$ \\
\hline$\rightarrow_{27}$ & $\begin{array}{l}\langle\max (\overline{\operatorname{sg}}(1-b), \operatorname{sg}(c)), \\
\min (\operatorname{sg}(a), \overline{\operatorname{sg}}(1-d))\rangle\end{array}$ \\
\hline$\rightarrow_{28}$ & $\langle\max (\overline{\operatorname{sg}}(1-b), c), \min (a, d)\rangle$ \\
\hline$\rightarrow_{29}$ & $\begin{array}{l}\langle\max (\overline{\operatorname{sg}}(1-b), \overline{\operatorname{sg}}(1-c)), \\
\min (a, \overline{\operatorname{sg}}(1-d))\rangle\end{array}$ \\
\hline$\rightarrow_{30}$ & $\langle\max (1-a, \min (a, 1-d)), \min (a, d)\rangle$ \\
\hline$\rightarrow_{31}$ & $\langle\overline{\operatorname{sg}}(a+d-1), d \cdot \operatorname{sg}(a+d-1)\rangle$ \\
\hline$\rightarrow_{32}$ & $\langle 1-d \cdot \operatorname{sg}(a+d-1), d \cdot \operatorname{sg}(a+d-1)\rangle$ \\
\hline$\rightarrow_{33}$ & $\langle 1-\min (a, d), \min (a, d)\rangle$ \\
\hline$\rightarrow_{34}$ & $\langle\min (1,2-a-d), \max (0, a+d-1)\rangle$ \\
\hline$\rightarrow 35$ & $\langle 1-a . d, a . d\rangle$ \\
\hline$\rightarrow_{36}$ & $\begin{array}{l}\langle\min (1-\min (a, d), \max (a, 1-a), \\
\max (1-d, d)), \max (\min (a, d), \\
\min (a, 1-a), \min (1-d, d))\rangle\end{array}$ \\
\hline$\rightarrow_{37}$ & $\begin{array}{l}\langle 1-\max (a, d) \cdot \operatorname{sg}(a+d-1) \\
\max (a, d) \cdot \operatorname{sg}(a+d-1)\rangle\end{array}$ \\
\hline$\rightarrow_{38}$ & $\left\langle 1-a+\left(a^{2} \cdot(1-d)\right), a \cdot(1-a)+a^{2} \cdot d\right\rangle$ \\
\hline$\rightarrow_{39}$ & $\begin{array}{l}\langle(1-d) \cdot \overline{\operatorname{sg}}(1-a)+\operatorname{sg}(1-a) \\
\cdot(\overline{\operatorname{sg}}(d)+(1-a) \cdot \operatorname{sg}(d)) \\
d . \overline{\operatorname{sg}}(1-a)+a \cdot \operatorname{sg}(1-a) \cdot \operatorname{sg}(d)\rangle\end{array}$ \\
\hline$\rightarrow_{40}$ & $\langle 1-\operatorname{sg}(a+d-1), 1-\overline{\operatorname{sg}}(a+d-1)\rangle$ \\
\hline$\rightarrow_{41}$ & $\langle\max (\overline{\operatorname{sg}}(a), 1-d), \min (\operatorname{sg}(a), d)\rangle$ \\
\hline$\rightarrow_{42}$ & $\begin{array}{l}\langle\max (\overline{\operatorname{sg}}(a), \operatorname{sg}(1-d)) \\
\min (\operatorname{sg}(a), \overline{\operatorname{sg}}(1-d))\rangle\end{array}$ \\
\hline$\rightarrow_{43}$ & $\langle\max (\overline{\operatorname{sg}}(a), 1-d), \min (\operatorname{sg}(a), d)\rangle$ \\
\hline$\rightarrow_{44}$ & $\langle\max (\overline{\operatorname{sg}}(a), 1-d), \min (a, d)\rangle$ \\
\hline$\rightarrow_{45}$ & $\langle\max (\overline{\operatorname{sg}}(a), \overline{\operatorname{sg}}(d)), \min (a, \overline{\operatorname{sg}}(1-d))\rangle$ \\
\hline$\rightarrow_{46}$ & $\langle\max (b, \min (1-b, c)), 1-\max (b, c)\rangle$ \\
\hline$\rightarrow_{47}$ & $\langle\overline{\operatorname{sg}}(1-b-c),(1-c) \cdot \operatorname{sg}(1-b-c)\rangle$ \\
\hline$\rightarrow_{48}$ & $\begin{array}{l}\langle 1-(1-c) \cdot \operatorname{sg}(1-b-c) \\
(1-c) \cdot \operatorname{sg}(1-b-c)\rangle\end{array}$ \\
\hline$\rightarrow_{49}$ & $\langle\min (1, b+c), \max (0,1-b-c)\rangle$ \\
\hline$\rightarrow_{50}$ & $\langle b+c-b \cdot c, 1-b-c+b . c\rangle$ \\
\hline$\rightarrow_{51}$ & $\begin{array}{l}\langle\min (\max (b, c), \max (1-b, b), \\
\max (c, 1-c)), \max (1-\max (b, c), \\
\min (1-b, b), \min (c, 1-c))\rangle\end{array}$ \\
\hline
\end{tabular}

\begin{tabular}{|c|c|}
\hline$\rightarrow_{52}$ & $\begin{array}{l}\langle 1-(1-\min (b, c)) \cdot \operatorname{sg}(1-b-c), \\
1-\min (b, c) \cdot \operatorname{sg}(1-b-c)\rangle\end{array}$ \\
\hline$\rightarrow_{53}$ & $\begin{array}{l}\left\langle b+(1-b)^{2} \cdot c\right. \\
\left.(1-b) \cdot b+(1-b)^{2} \cdot(1-c)\right\rangle\end{array}$ \\
\hline$\rightarrow_{54}$ & $\begin{array}{l}\langle c \cdot \overline{\mathrm{sg}}(b)+\operatorname{sg}(b) \cdot(\overline{\operatorname{sg}}(1-c)+b \cdot \operatorname{sg}(1-c)), \\
(1-c) \cdot \overline{\operatorname{sg}}(b)+(1-b) \cdot \operatorname{sg}(b) \cdot \operatorname{sg}(1-c)\rangle\end{array}$ \\
\hline$\rightarrow_{55}$ & $\langle 1-\operatorname{sg}(1-b-c), 1-\overline{\operatorname{sg}}(1-b-c)\rangle$ \\
\hline$\rightarrow_{56}$ & $\begin{array}{l}\langle\max (\overline{\operatorname{sg}}(1-b), c), \\
\min (\operatorname{sg}(1-b),(1-c))\rangle\end{array}$ \\
\hline$\rightarrow_{57}$ & $\begin{array}{l}\langle\max (\overline{\operatorname{sg}}(1-b), \\
\operatorname{sg}(c)), \min (\operatorname{sg}(1-b), \overline{\operatorname{sg}}(c))\rangle\end{array}$ \\
\hline$\rightarrow_{58}$ & $\langle\max (\overline{\operatorname{sg}}(1-b), \overline{\operatorname{sg}}(1-c)), 1-\max (b, c)\rangle$ \\
\hline$\rightarrow_{59}$ & $\langle\max (\overline{\operatorname{sg}}(1-b), c),(1-\max (b, c))\rangle$ \\
\hline$\rightarrow_{60}$ & $\begin{array}{l}\langle\max (\overline{\operatorname{sg}}(1-b), \overline{\operatorname{sg}}(1-c)) \\
\min ((1-b), \overline{\operatorname{sg}}(c))\rangle\end{array}$ \\
\hline$\rightarrow_{61}$ & $\langle\max (c, \min (b, d)), \min (a, d)\rangle$ \\
\hline$\rightarrow_{62}$ & $\langle\overline{\operatorname{sg}}(d-b), a \cdot \operatorname{sg}(d-b)\rangle$ \\
\hline$\rightarrow_{63}$ & $\langle 1-(1-b) \cdot \operatorname{sg}(d-b), a \cdot \operatorname{sg}(d-b)\rangle$ \\
\hline$\rightarrow_{64}$ & $\langle c+b . d, a . d\rangle$ \\
\hline$\rightarrow_{65}$ & $\begin{array}{l}\langle 1-(1-\min (b, c)) \cdot \operatorname{sg}(d-b), \\
\max (a, d) \cdot \operatorname{sg}(d-b) \cdot \operatorname{sg}(a-c)\rangle\end{array}$ \\
\hline$\rightarrow_{66}$ & $\left\langle c+d^{2} . b, b . d+d^{2} . a\right\rangle$ \\
\hline$\rightarrow_{67}$ & $\begin{array}{l}\langle b \cdot \overline{\operatorname{sg}}(1-d)+\operatorname{sg}(1-d) \cdot(\overline{\operatorname{sg}}(1-b) \\
+c \cdot \operatorname{sg}(1-b)), \\
a \cdot \overline{\operatorname{sg}}(1-d)+d \cdot \operatorname{sg}(1-d) \cdot \operatorname{sg}(1-b)\rangle\end{array}$ \\
\hline$\rightarrow_{68}$ & $\langle 1-(1-b) \cdot \operatorname{sg}(d-b), a \cdot \operatorname{sg}(d-b) \cdot \operatorname{sg}(a-c)\rangle$ \\
\hline$\rightarrow_{69}$ & $\begin{array}{l}\langle 1-(1-b) \cdot \operatorname{sg}(d-b)-a \cdot \overline{\operatorname{sg}}(d-b) \cdot \operatorname{sg}(a-c) \\
a \cdot \operatorname{sg}(a-c)\rangle\end{array}$ \\
\hline$\rightarrow_{70}$ & $\langle\max (\overline{\operatorname{sg}}(d), b), \min (\operatorname{sg}(d), a)\rangle$ \\
\hline$\rightarrow_{71}$ & $\left\langle\max (b, c), \min \left(c . d+d^{2}, a\right)\right\rangle$ \\
\hline$\rightarrow_{72}$ & $\langle\max (b, c), \min (1-c, a)\rangle$ \\
\hline$\rightarrow_{73}$ & $\begin{array}{l}\langle\max (1-\max (\operatorname{sg}(d), \operatorname{sg}(1-c)), b), \\
\min (\operatorname{sg}(1-c), a)\rangle\end{array}$ \\
\hline$\rightarrow_{74}$ & $\langle\max (\operatorname{sg}(b), \overline{\operatorname{sg}}(d)), \min (\overline{\operatorname{sg}}(b), \operatorname{sg}(d))\rangle$ \\
\hline$\rightarrow_{75}$ & $\begin{array}{l}\langle\max (c, b \cdot(a+b)), \min (d .(c+d), \\
\left.\left.a .\left(b^{2}+a\right)+a \cdot b\right)\right\rangle\end{array}$ \\
\hline$\rightarrow_{76}$ & $\langle\max (c, 1-a), \min (1-c, a)\rangle$ \\
\hline$\rightarrow 77$ & $\begin{array}{l}\langle(1-\min (\overline{\operatorname{sg}}(1-a), \operatorname{sg}(1-c))), \\
\min (\overline{\operatorname{sg}}(1-a), \operatorname{sg}(1-c))\rangle\end{array}$ \\
\hline$\rightarrow 78$ & $\langle\max (\overline{\operatorname{sg}}(1-c), b), \min (\operatorname{sg}(d), a)\rangle$ \\
\hline$\rightarrow_{79}$ & $\begin{array}{l}\langle\max (\overline{\operatorname{sg}}(1-c), \operatorname{sg}(b)) \\
\min (\operatorname{sg}(d), \overline{\operatorname{sg}}(1-a))\rangle\end{array}$ \\
\hline$\rightarrow_{80}$ & $\langle\max (\overline{\operatorname{sg}}(1-c), b), \min (d, a)\rangle$ \\
\hline$\rightarrow_{81}$ & $\begin{array}{l}\langle\max (\overline{\operatorname{sg}}(1-b), \overline{\operatorname{sg}}(1-c)), \\
\min (d, \overline{\operatorname{sg}}(1-a))\rangle\end{array}$ \\
\hline$\rightarrow_{82}$ & $\langle\max (1-d, \min (d, 1-a)), \min (d, a)\rangle$ \\
\hline$\rightarrow_{83}$ & $\langle\overline{\operatorname{sg}}(a+d-1), a \cdot \operatorname{sg}(a+d-1)\rangle$ \\
\hline$\rightarrow_{84}$ & $\langle 1-a \cdot \operatorname{sg}(a+d+1), a \cdot \operatorname{sg}(a+d+1)\rangle$ \\
\hline$\rightarrow_{85}$ & $\left\langle 1-d+d^{2} \cdot(1-a), d .(1-d)+d^{2}.\right\rangle$ \\
\hline$\rightarrow_{86}$ & $\begin{array}{l}\langle(1-a) \cdot \overline{\operatorname{sg}}(1-d)+\operatorname{sg}(1-d) \\
. \overline{\operatorname{sg}}(a+\min (1-d, \operatorname{sg}(a))) \\
a \cdot \overline{\operatorname{sg}}(1-d)+d \cdot \operatorname{sg}(1-d) \cdot \operatorname{sg}(a)\rangle\end{array}$ \\
\hline$\rightarrow_{87}$ & $\langle\max (\overline{\operatorname{sg}}(d), 1-a), \min (\operatorname{sg}(d), a)\rangle$ \\
\hline$\rightarrow_{88}$ & $\begin{array}{l}\langle\max (\overline{\operatorname{sg}}(d), \operatorname{sg}(1-a)), \\
\min (\operatorname{sg}(d), \overline{\operatorname{sg}}(1-a))\rangle\end{array}$ \\
\hline$\rightarrow_{89}$ & $\langle\max (\overline{\operatorname{sg}}(d), 1-a), \min (d, a)\rangle$ \\
\hline$\rightarrow 90$ & $\langle\max (\overline{\operatorname{sg}}(a), \overline{\operatorname{sg}}(d)), \min (d, \overline{\operatorname{sg}}(1-a))\rangle$ \\
\hline$\rightarrow_{91}$ & $\langle\max (c, \min (1-c, b)), 1-\max (b, c)\rangle$ \\
\hline$\rightarrow_{92}$ & $\langle\overline{\operatorname{sg}}(1-b-c), \min (1-b, \operatorname{sg}(1-b-c))\rangle$ \\
\hline
\end{tabular}




\begin{tabular}{|c|c|}
\hline$\rightarrow_{93}$ & $\begin{array}{l}\langle(1-\min (1-b, \operatorname{sg}(1-b-c)), \\
\min (1-b, \operatorname{sg}(1-b-c))\rangle\end{array}$ \\
\hline$\rightarrow_{94}$ & $\left\langle c+(1-c)^{2} \cdot b,(1-c) \cdot c+(1-c)^{2} \cdot(1-b)\right\rangle$ \\
\hline$\rightarrow_{95}$ & $\begin{array}{l}\langle\min (b, \overline{\operatorname{sg}}(c))+\operatorname{sg}(c) \cdot(\overline{\operatorname{sg}}(1-b) \\
+\min (c, \operatorname{sg}(1-b))),(\min (1-b, \overline{\operatorname{sg}}(c)) \\
+\min (1-c, \operatorname{sg}(c), \operatorname{sg}(1-b))\rangle\end{array}$ \\
\hline$\rightarrow_{96}$ & $\langle\max (\overline{\mathrm{sg}}(1-c), b), \min (\operatorname{sg}(1-b), 1-c)\rangle$ \\
\hline$\rightarrow_{97}$ & $\begin{array}{l}\langle\max (\overline{\operatorname{sg}}(1-c), \operatorname{sg}(b)), \\
\min (\operatorname{sg}(1-c), \overline{\operatorname{sg}}(b))\rangle\end{array}$ \\
\hline$\rightarrow_{98}$ & $\langle\max (\overline{\mathrm{sg}}(1-c), b), 1-\max (b, c)\rangle$ \\
\hline$\rightarrow_{99}$ & $\begin{array}{l}\langle\max (\overline{\operatorname{sg}}(1-c), \overline{\operatorname{sg}}(1-b)), \\
\min (1-c, \overline{\operatorname{sg}}(b))\rangle\end{array}$ \\
\hline$\rightarrow_{100}$ & $\begin{array}{l}\langle\max (\min (b, \operatorname{sg}(a)), c), \\
\min (a, \operatorname{sg}(b), d)\rangle\end{array}$ \\
\hline$\rightarrow_{101}$ & $\begin{array}{l}\langle\max (\min (b, \operatorname{sg}(a)), \min (c, \operatorname{sg}(d))), \\
\min (a, \operatorname{sg}(b), \operatorname{sg}(c), d)\rangle\end{array}$ \\
\hline$\rightarrow_{102}$ & $\langle\max (b, \min (c, \operatorname{sg}(d))), \min (a, \operatorname{sg}(c), d)\rangle$ \\
\hline$\rightarrow_{103}$ & $\begin{array}{l}\langle\max (\min (1-a, \operatorname{sg}(a)), 1-d), \\
\min (a, \operatorname{sg}(1-a), d)\rangle\end{array}$ \\
\hline$\rightarrow_{104}$ & $\begin{array}{l}\langle\max (\min (1-a, \operatorname{sg}(a)) \\
\min (1-d, \operatorname{sg}(d))), \\
\min (a, \operatorname{sg}(1-a), d, \operatorname{sg}(1-d))\rangle\end{array}$ \\
\hline$\rightarrow_{105}$ & $\begin{array}{l}\langle\max (1-a, \min (1-d, \operatorname{sg}(d))), \\
\min (a, d, \operatorname{sg}(1-d))\rangle\end{array}$ \\
\hline$\rightarrow_{106}$ & $\begin{array}{l}\langle\max (\min (b, \operatorname{sg}(1-b)), c), \\
\min (1-b, \operatorname{sg}(b), 1-c)\rangle\end{array}$ \\
\hline$\rightarrow_{107}$ & $\begin{array}{l}\langle\max (\min (b, \operatorname{sg}(1-b)) \\
\min (c, \operatorname{sg}(1-c))) \\
\min (1-b, \operatorname{sg}(b), 1-c, \operatorname{sg}(c))\rangle\end{array}$ \\
\hline$\rightarrow_{108}$ & $\begin{array}{l}\langle\max (b, \min (c, \operatorname{sg}(1-c))), \\
\min (1-b, 1-c, \operatorname{sg}(c))\rangle\end{array}$ \\
\hline$\rightarrow_{109}$ & $\begin{array}{l}\langle b+\min (\overline{\operatorname{sg}}(1-a), c), \\
a . b+\min (\overline{\operatorname{sg}}(1-a), d))\rangle\end{array}$ \\
\hline$\rightarrow_{110}$ & $\langle\max (b, c), \min (a \cdot b+\overline{\operatorname{sg}}(1-a), d)\rangle$ \\
\hline$\rightarrow_{111}$ & $\begin{array}{l}\langle\max (b, c . d+\overline{\operatorname{sg}}(1-c)) \\
\min (a . b+\overline{\operatorname{sg}}(1-a), d .(c . d+\overline{\operatorname{sg}}(1-c)) \\
+\overline{\operatorname{sg}}(1-d))\rangle\end{array}$ \\
\hline$\rightarrow_{112}$ & $\langle b+c-b \cdot c, a \cdot b+\overline{\operatorname{sg}}(1-a) \cdot d\rangle$ \\
\hline$\rightarrow_{113}$ & $\begin{array}{l}\langle b+c \cdot d-b \cdot(c \cdot d+\overline{\mathrm{sg}}(1-c)), \\
(a \cdot b+\overline{\operatorname{sg}}(1-a)) \cdot(d \cdot(c \cdot d+\overline{\operatorname{sg}}(1-c)) \\
+\overline{\operatorname{sg}}(1-d))\rangle\end{array}$ \\
\hline$\rightarrow_{114}$ & $\begin{array}{l}\langle 1-a+\min (\overline{\operatorname{sg}}(1-a), 1-d), \\
a .(1-a)+\min (\overline{\operatorname{sg}}(1-a), d)\rangle\end{array}$ \\
\hline$\rightarrow_{115}$ & $\begin{array}{l}\langle 1-\min (a, d), \\
\min (a .(1-a)+\overline{\operatorname{sg}}(1-a), d)\rangle\end{array}$ \\
\hline$\rightarrow_{116}$ & $\begin{array}{l}\langle\max (1-a,(1-d) \cdot d+\overline{\operatorname{sg}}(d)) \\
\min (a \cdot(1-a)+\overline{\operatorname{sg}}(1-a), d \cdot((1-d) . d \\
+\overline{\operatorname{sg}}(d))+\overline{\operatorname{sg}}(1-d))\rangle\end{array}$ \\
\hline$\rightarrow_{117}$ & $\begin{array}{l}\langle 1-a-d+a . d, \\
(a .(1-a)+\overline{\operatorname{sg}}(1-a)) . d\rangle\end{array}$ \\
\hline$\rightarrow_{118}$ & $\begin{array}{l}\langle 1-a+(1-d) \cdot d-(1-a) \cdot((1-d) \\
. d+\overline{\operatorname{sg}}(d)),(a \cdot(1-a)+\overline{\operatorname{sg}}(1-a)) \\
. d \cdot((1-d) \cdot d+\overline{\operatorname{sg}}(d))+\overline{\operatorname{sg}}(1-d)\rangle\end{array}$ \\
\hline$\rightarrow_{119}$ & $\begin{array}{l}\langle b+\min (\overline{\operatorname{sg}}(b), c), \\
(1-b) \cdot b+\min (\overline{\operatorname{sg}}(b), 1-c)\rangle\end{array}$ \\
\hline$\rightarrow_{120}$ & $\langle\max (b, c), \min ((1-b) \cdot b+\overline{\operatorname{sg}}(b), 1-c)\rangle$ \\
\hline$\rightarrow_{121}$ & $\begin{array}{l}\langle\max (b, c \cdot(1-c)+\overline{\operatorname{sg}}(1-c)) \\
\min ((1-b) \cdot b+\overline{\operatorname{sg}}(b),(1-c) \cdot(c \cdot(1-c) \\
+\overline{\operatorname{sg}}(1-c)))+\overline{\operatorname{sg}}(c)\rangle\end{array}$ \\
\hline$\rightarrow_{122}$ & $\langle b+c-b \cdot c,((1-c) \cdot b+\overline{\operatorname{sg}}(b)) \cdot(1-c)\rangle$ \\
\hline
\end{tabular}

\begin{tabular}{|c|c|}
\hline$\rightarrow_{123}$ & $\begin{array}{l}\langle b+c \cdot(1-c)-(b .(c .(1-c)+\overline{\operatorname{sg}}(1-c))), \\
((1-b) \cdot b+\overline{\operatorname{sg}}(b)) \cdot(((1-c) \\
.(c .(1-c)+\overline{\operatorname{sg}}(1-c)))+\overline{\operatorname{sg}}(c))\rangle\end{array}$ \\
\hline$\rightarrow_{124}$ & $\begin{array}{l}\langle c+\min (\overline{\operatorname{sg}}(1-d), b), \\
c . d+\min (\overline{\operatorname{sg}}(1-d), a)\rangle\end{array}$ \\
\hline$\rightarrow_{125}$ & $\langle\max (b, c), \min (c . d+\overline{\operatorname{sg}}(1-d), a)\rangle$ \\
\hline$\rightarrow_{126}$ & $\begin{array}{l}\langle\max (c, a \cdot b+\overline{\operatorname{sg}}(1-b)) \\
\min (c . d+\overline{\operatorname{sg}}(1-d), a \cdot(a . b \\
+\overline{\operatorname{sg}}(1-b))+\overline{\operatorname{sg}}(1-a))\rangle\end{array}$ \\
\hline$\rightarrow_{127}$ & $\langle b+c-b . c,(c . d+\overline{\operatorname{sg}}(1-d)) \cdot a\rangle$ \\
\hline$\rightarrow_{128}$ & $\begin{array}{l}\langle c+a \cdot b-c \cdot(a \cdot b+\overline{\operatorname{sg}}(1-b)) \\
(c \cdot d+\overline{\operatorname{sg}}(1-d)) \cdot(a \cdot(a \cdot b+\overline{\operatorname{sg}}(1-b)) \\
+\overline{\operatorname{sg}}(1-a))\rangle\end{array}$ \\
\hline$\rightarrow_{129}$ & $\begin{array}{l}\langle 1-d+\min (\overline{\operatorname{sg}}(1-d), 1-a), \\
d .(1-d)+\min (\overline{\operatorname{sg}}(1-d), a)\rangle\end{array}$ \\
\hline$\rightarrow_{130}$ & $\begin{array}{l}\langle 1-\min (d, a) \\
\min (d .(1-d)+\overline{\operatorname{sg}}(1-d), a)\rangle\end{array}$ \\
\hline$\rightarrow_{131}$ & $\begin{array}{l}\langle\max (1-d,(1-a) \cdot a+\overline{\operatorname{sg}}(a)), \\
\min (d .(1-d)+\overline{\operatorname{sg}}(1-d), \\
a .((1-a) \cdot a+\overline{\operatorname{sg}}(a))+\overline{\operatorname{sg}}(1-a))\rangle\end{array}$ \\
\hline$\rightarrow_{132}$ & $\langle 1-a . d,(d .(1-d)+\overline{\operatorname{sg}}(1-d)) . a\rangle$ \\
\hline$\rightarrow_{133}$ & $\begin{array}{l}\langle 1-d+(1-a) \cdot a-(1-d) \\
.((1-a) \cdot a+\overline{\operatorname{sg}}(a)) \\
(d .(1-d)+\overline{\operatorname{sg}}(1-d)) \\
.(a .((1-a) \cdot a+\overline{\operatorname{sg}}(a))+\overline{\operatorname{sg}}(1-a))\rangle\end{array}$ \\
\hline$\rightarrow_{134}$ & $\begin{array}{l}\langle c+\min (\overline{\operatorname{sg}}(c), b) \\
(1-c) \cdot c+\min (\overline{\operatorname{sg}}(c),(1-b))\rangle\end{array}$ \\
\hline$\rightarrow_{135}$ & $\begin{array}{l}\langle\max (b, c), \\
\min ((1-c) \cdot c+\overline{\operatorname{sg}}(c), 1-b)\rangle\end{array}$ \\
\hline$\rightarrow_{136}$ & $\begin{array}{l}\langle\max (c,(b \cdot(1-b)+\overline{\operatorname{sg}}(1-b))), \\
\min ((1-c) \cdot c+\overline{\operatorname{sg}}(c), \\
(1-b) \cdot(b \cdot(1-b)+\overline{\operatorname{sg}}(1-b)) \\
+\overline{\operatorname{sg}}(b))\rangle\end{array}$ \\
\hline$\rightarrow_{137}$ & $\begin{array}{l}\langle b+c-b \cdot c, \\
((1-c) \cdot c+\overline{\operatorname{sg}}(c)) \cdot(1-b)\rangle\end{array}$ \\
\hline$\rightarrow_{138}$ & $\begin{array}{l}\langle c+b \cdot(1-b)-c .(b \cdot(1-b)+\overline{\operatorname{sg}}(1-b)) \\
((1-c) \cdot c+\overline{\operatorname{sg}}(c)) \cdot((1-b) \\
.(b .(1-b)+\overline{\operatorname{sg}}(1-b))+\overline{\operatorname{sg}}(b))\rangle\end{array}$ \\
\hline$\rightarrow_{139}$ & $\left\langle\frac{b+c}{2}, \frac{a+d}{2}\right\rangle$ \\
\hline$\rightarrow_{140}$ & $\left\langle\frac{b+c+\min (b, c)}{3}, \frac{a+d+\max (a, d)}{3}\right\rangle$ \\
\hline$\rightarrow_{141}$ & $\left\langle\frac{b+c+\max (b, c)}{3}, \frac{a+d+\min (a, d)}{3}\right\rangle$ \\
\hline$\rightarrow_{142}$ & $\left\langle\frac{3-a-d-\max (a, d)}{3}, \frac{a+d+\max (a, d)}{3}\right\rangle$ \\
\hline$\rightarrow_{143}$ & $\left\langle\frac{1-a+c+\min (1-a, c)}{3}, \frac{2+a-c-\min (1-a, c)}{3}\right\rangle$ \\
\hline$\rightarrow_{144}$ & $\left\langle\frac{1+b-d+\min (b, 1-d)}{3}, \frac{2-b+d+\min (b, 1-d)}{3}\right\rangle$ \\
\hline$\rightarrow_{145}$ & $\left\langle\frac{b+c+\min (b, c)}{3}, \frac{3-b-c-\min (b, c)}{3}\right\rangle$ \\
\hline$\rightarrow_{146}$ & $\left\langle\frac{3-a-d-\min (a, d)}{3}, \frac{a+d+\min (a, d)}{3}\right\rangle$ \\
\hline$\rightarrow_{147}$ & $\left\langle\frac{1-a+c+\max (1-a, c)}{3}, \frac{2+a-c-\max (1-a, c)}{3}\right\rangle$ \\
\hline$\rightarrow_{148}$ & $\left\langle\frac{1+b-d+\max (b, 1-d)}{3}, \frac{2-b+d-\max (b, 1-d)}{3}\right\rangle$ \\
\hline$\rightarrow_{149}$ & $\left\langle\frac{b+c+\max (b, c)}{3}, \frac{3-b-c-\max (b, c)}{3}\right.$ \\
\hline$\rightarrow_{150, \lambda}$ & $\left\langle\frac{b+c+\lambda-1}{2 \lambda}, \frac{a+d+\lambda-1}{2 \lambda}\right.$, where $\lambda \geq 1$ \\
\hline$\rightarrow_{151, \gamma}$ & $\left\langle\frac{b+c+\gamma}{2 \gamma+1}, \frac{a+d+\gamma-1}{2 \gamma+1}\right.$, where $\gamma \geq 1$ \\
\hline$\rightarrow_{152, \alpha, \beta}$ & $\begin{array}{l}\left\langle\frac{b+c+\alpha-1}{\alpha+\beta}, \frac{a+d+\beta-1}{\alpha+\beta},\right. \\
\text { where } \alpha \geq 1, \beta \in[0, \alpha]\end{array}$ \\
\hline$\rightarrow_{153, \varepsilon, \eta}$ & $\begin{array}{l}\langle\min (1, \max (c, b+\varepsilon)), \\
\max (0, \min (d, a-\eta))\rangle \\
\text { where } \varepsilon, \eta \in[0,1] \text { and } \varepsilon \leq \eta\end{array}$ \\
\hline
\end{tabular}


Table 2

\begin{tabular}{|c|c|}
\hline$\neg 1$ & $\langle b, a\rangle$ \\
\hline$\neg 2$ & $\langle\overline{\operatorname{sg}}(a), \operatorname{sg}(a)\rangle$ \\
\hline$\neg 3$ & $\left\langle b, a \cdot b+a^{2}\right\rangle$ \\
\hline$\neg 4$ & $\langle b, 1-b\rangle$ \\
\hline$\neg_{5}$ & $\langle\overline{\mathrm{sg}}(1-b), \operatorname{sg}(1-b)\rangle$ \\
\hline$\neg 6$ & $\langle\overline{\mathrm{sg}}(1-b), \operatorname{sg}(a)\rangle$ \\
\hline$\neg 7$ & $\langle\overline{\mathrm{sg}}(1-b), a\rangle$ \\
\hline$\neg 8$ & $\langle 1-a, a\rangle$ \\
\hline$\neg 9$ & $\langle\overline{\operatorname{sg}}(a), a\rangle$ \\
\hline$\neg 10$ & $\langle\overline{\mathrm{sg}}(1-b), 1-b\rangle$ \\
\hline$\neg 11$ & $\langle\operatorname{sg}(b), \overline{\operatorname{sg}}(b)\rangle$ \\
\hline$\neg 12$ & $\left\langle b \cdot(b+a), \min \left(1, a \cdot\left(b^{2}+a+b \cdot a\right)\right)\right\rangle$ \\
\hline$\neg 13$ & $\langle\operatorname{sg}(1-a), \overline{\operatorname{sg}}(1-a)\rangle$ \\
\hline$\neg 14$ & $\langle\operatorname{sg}(b), \overline{\operatorname{sg}}(1-a)\rangle$ \\
\hline$\neg 15$ & $\langle\overline{\mathrm{sg}}(1-b), \overline{\mathrm{sg}}(1-a)\rangle$ \\
\hline$\neg 16$ & $\langle\overline{\operatorname{sg}}(a), \overline{\operatorname{sg}}(1-a)\rangle$ \\
\hline$\neg 17$ & $\langle\overline{\mathrm{sg}}(1-b), \overline{\mathrm{sg}}(b)\rangle$ \\
\hline$\neg 18$ & $\langle b . \operatorname{sg}(a), a . \operatorname{sg}(b)\rangle$ \\
\hline$\neg 19$ & $\langle b . \operatorname{sg}(a), 0\rangle$ \\
\hline$\neg 20$ & $\langle b, 0\rangle$ \\
\hline$\neg 21$ & $\langle\min (1-a, \operatorname{sg}(a)), \min (a, \operatorname{sg}(1-a))\rangle$ \\
\hline$\neg 22$ & $\langle\min (1-a, \operatorname{sg}(a)), 0\rangle$ \\
\hline$\neg 23$ & $\langle 1-a, 0\rangle$ \\
\hline$\neg 24$ & $\langle\min (b, \operatorname{sg}(1-b)), \min (1-b, \operatorname{sg}(b))\rangle$ \\
\hline$\neg 25$ & $\langle\min (b, \operatorname{sg}(1-b)), 0\rangle$ \\
\hline$\neg_{26}$ & $\langle b, a \cdot b+\overline{\mathrm{sg}}(1-a)\rangle$ \\
\hline$\neg_{27}$ & $\langle 1-a, a .(1-a)+\overline{\operatorname{sg}}(1-a)\rangle$ \\
\hline$\neg 28$ & $\langle b,(1-b) \cdot b+\overline{\operatorname{sg}}(b)\rangle$ \\
\hline$\neg 29$ & $\begin{array}{l}\langle\max (0, b \cdot a+\overline{\operatorname{sg}}(1-b)) \\
\min (1, a \cdot(b \cdot a+\overline{\operatorname{sg}}(1-b))+\overline{\operatorname{sg}}(1-a))\rangle\end{array}$ \\
\hline$\neg 30$ & $\begin{array}{l}\langle a . b, a \cdot(a . b+\overline{\operatorname{sg}}(1-b)) \\
+\overline{\operatorname{sg}}(1-a)\rangle\end{array}$ \\
\hline$\neg 31$ & $\begin{array}{l}\langle\max (0,(1-a) \cdot a+\overline{\operatorname{sg}}(a)) \\
\min (1, a \cdot((1-a) \cdot a+\overline{\operatorname{sg}}(a)) \\
+\overline{\operatorname{sg}}(1-a))\rangle\end{array}$ \\
\hline$\neg 32$ & $\begin{array}{l}\langle(1-a) \cdot a, a \cdot((1-a) \cdot a \\
+\overline{\operatorname{sg}}(a))+\overline{\operatorname{sg}}(1-a)\rangle\end{array}$ \\
\hline$\neg 33$ & $\begin{array}{l}\langle b \cdot(1-b)+\overline{\mathrm{sg}}(1-b) \\
(1-b) \cdot(b \cdot(1-b)+\overline{\mathrm{sg}}(1-b))+\overline{\mathrm{sg}}(b))\rangle\end{array}$ \\
\hline$\neg 34$ & $\begin{array}{l}\langle b \cdot(1-b),(1-b) \cdot(b \cdot(1-b) \\
+\overline{\operatorname{sg}}(1-b))+\overline{\operatorname{sg}}(b)\rangle\end{array}$ \\
\hline$\neg 35$ & $\left\langle\frac{b}{2}, \frac{1+a}{2}\right\rangle$ \\
\hline$\neg 36$ & $\left\langle\frac{b}{3}, \frac{2+a}{3}\right\rangle$ \\
\hline$\neg 37$ & $\left\langle\frac{2 b}{3}, \frac{2 a+1}{3}\right\rangle$ \\
\hline$\neg 38$ & $\left\langle\frac{1-a}{3}, \frac{2+a}{3}\right\rangle$ \\
\hline$\neg 39$ & $\left\langle\frac{b}{3}, \frac{3-b}{3}\right\rangle$ \\
\hline$\neg 40$ & $\left\langle\frac{2-2 a}{3}, \frac{1+2 a}{3}\right\rangle$ \\
\hline$\neg 41$ & $\left\langle\frac{2 b}{3}, \frac{3-2 b}{3}\right\rangle$ \\
\hline$\neg 42, \lambda$ & $\left\langle\frac{b+\lambda-1}{2 \lambda}, \frac{a+\lambda}{2 \lambda}\right.$, where $\lambda \geq 1$ \\
\hline$\neg 43, \gamma$ & $\left\langle\frac{b+\gamma}{2 \gamma+1}, \frac{a+\gamma}{2 \gamma+1}\right.$, where $\gamma \geq 1$ \\
\hline$\neg 44, \alpha, \beta$ & $\left\langle\frac{b+\alpha-1}{\alpha+\beta}, \frac{a+\beta}{\alpha+\beta}\right.$, where $\alpha \geq 1, \beta \in[0, \alpha]$ \\
\hline$\neg 45, \varepsilon, \eta$ & $\begin{array}{l}\langle\min (1, b+\varepsilon), \max (0, a-\eta)\rangle \\
\text { where } \varepsilon, \eta \in[0,1] \text { and } \varepsilon \leq \eta\end{array}$ \\
\hline
\end{tabular}

The relations between the implications and negations are shown in Table 3.
Table 3

\begin{tabular}{|c|c|}
\hline$\neg 1$ & $\begin{array}{l}\rightarrow_{1}, \rightarrow_{4}, \rightarrow_{5}, \rightarrow_{6}, \rightarrow_{7}, \rightarrow_{10}, \rightarrow_{13}, \rightarrow_{61}, \\
\rightarrow_{63}, \rightarrow_{64}, \rightarrow_{66}, \rightarrow_{67}, \rightarrow_{68}, \rightarrow_{69}, \rightarrow_{70}, \rightarrow_{71}, \\
\rightarrow_{72}, \rightarrow_{73}, \rightarrow_{78}, \rightarrow_{80}, \rightarrow_{124}, \rightarrow_{125}, \rightarrow_{127}\end{array}$ \\
\hline$\neg_{2}$ & $\begin{array}{l}\rightarrow_{2}, \rightarrow_{3}, \rightarrow_{8}, \rightarrow_{11}, \rightarrow_{16}, \rightarrow_{20}, \rightarrow_{31}, \rightarrow_{32}, \\
\rightarrow_{37}, \rightarrow_{40}, \rightarrow_{41}, \rightarrow_{42}\end{array}$ \\
\hline$\neg 3$ & $\rightarrow_{9}, \rightarrow_{17}, \rightarrow_{21}$ \\
\hline$\neg 4$ & $\begin{array}{l}\rightarrow_{12}, \rightarrow_{18}, \rightarrow_{22}, \rightarrow_{46}, \rightarrow_{49}, \rightarrow_{50}, \rightarrow_{51}, \rightarrow_{53}, \\
\rightarrow_{54}, \rightarrow_{91}, \rightarrow_{93}, \rightarrow_{94}, \rightarrow_{95}, \rightarrow_{96}, \rightarrow_{98}, \rightarrow_{134}, \\
\rightarrow_{135}, \rightarrow_{137}\end{array}$ \\
\hline$\neg 5$ & $\begin{array}{l}\rightarrow_{14}, \rightarrow_{15}, \rightarrow_{19}, \rightarrow_{23}, \rightarrow_{47}, \rightarrow_{48}, \rightarrow_{52}, \rightarrow_{55} \\
\rightarrow_{56}, \rightarrow_{57}\end{array}$ \\
\hline$\neg 6$ & $\rightarrow_{24}, \rightarrow_{26}, \rightarrow_{27}, \rightarrow_{65}$ \\
\hline$\neg 7$ & $\rightarrow_{25}, \rightarrow_{28}, \rightarrow_{29}, \rightarrow_{62}$ \\
\hline$\neg 8$ & $\begin{array}{l}\rightarrow_{30}, \rightarrow_{33}, \rightarrow_{34}, \rightarrow_{35}, \rightarrow_{36}, \rightarrow_{38}, \rightarrow_{39}, \rightarrow_{76} \\
\rightarrow_{82}, \rightarrow_{84}, \rightarrow_{85}, \rightarrow_{86}, \rightarrow_{87}, \rightarrow_{89}, \rightarrow_{129} \\
\rightarrow_{130}, \rightarrow_{132}\end{array}$ \\
\hline$\neg 9$ & $\rightarrow_{43}, \rightarrow_{44}, \rightarrow_{45}, \rightarrow_{83}$ \\
\hline$\neg 10$ & $\rightarrow_{58}, \rightarrow_{59}, \rightarrow_{60}, \rightarrow_{92}$ \\
\hline$\neg 11$ & $\rightarrow_{74}, \rightarrow_{97}$ \\
\hline$\neg 12$ & $\rightarrow_{75}$ \\
\hline$\neg 13$ & $\rightarrow_{77}, \rightarrow_{88}$ \\
\hline$\neg 14$ & $\rightarrow 79$ \\
\hline$\neg 15$ & $\rightarrow_{81}$ \\
\hline$\neg 16$ & $\rightarrow 90$ \\
\hline$\neg 17$ & $\rightarrow 99$ \\
\hline$\neg 18$ & $\rightarrow_{100}$ \\
\hline$\neg 19$ & $\rightarrow_{101}$ \\
\hline$\neg 20$ & $\rightarrow_{102}, \rightarrow_{108}$ \\
\hline$\neg 21$ & $\rightarrow_{103}$ \\
\hline$\neg 22$ & $\rightarrow_{104}$ \\
\hline$\neg 23$ & $\rightarrow_{105}$ \\
\hline$\neg 24$ & $\rightarrow_{106}$ \\
\hline$\neg 25$ & $\rightarrow_{107}$ \\
\hline$\neg 26$ & $\rightarrow_{109}, \rightarrow_{110}, \rightarrow_{111}, \rightarrow_{112}, \rightarrow_{113}$ \\
\hline$\neg 27$ & $\rightarrow_{114}, \rightarrow_{115}, \rightarrow_{116}, \rightarrow_{117}, \rightarrow_{118}$ \\
\hline$\neg 28$ & $\rightarrow_{119}, \rightarrow_{120}, \rightarrow_{121}, \rightarrow_{122}, \rightarrow_{123}$ \\
\hline$\neg 29$ & $\rightarrow_{126}$ \\
\hline$\neg 30$ & $\rightarrow_{128}$ \\
\hline$\neg 31$ & $\rightarrow_{131}$ \\
\hline$\neg 32$ & $\rightarrow_{133}$ \\
\hline$\neg 33$ & $\rightarrow_{136}$ \\
\hline$\neg 34$ & $\rightarrow_{138}$ \\
\hline$\neg 35$ & $\rightarrow_{139}$ \\
\hline$\neg 36$ & $\rightarrow_{140}$ \\
\hline$\neg 37$ & $\rightarrow_{141}$ \\
\hline$\neg 38$ & $\rightarrow_{142}, \rightarrow_{143}$ \\
\hline$\neg 39$ & $\rightarrow_{144}, \rightarrow_{145}$ \\
\hline$\neg 40$ & $\rightarrow_{146}, \rightarrow_{147}$ \\
\hline$\neg 41$ & $\rightarrow_{148}, \rightarrow_{149}$ \\
\hline$\neg 42$ & $\rightarrow_{150}$ \\
\hline$\neg 43$ & $\rightarrow_{151}$ \\
\hline$\neg 44$ & $\rightarrow_{152}$ \\
\hline$\neg 45$ & $\rightarrow_{153}$ \\
\hline
\end{tabular}


3. Intutionistic fuzzy implications and the axioms of intuitionistic logic

First, we mention that the last four from the above intutionistic fuzzy implications $\left(\rightarrow_{150, \lambda}, \rightarrow_{151, \gamma}\right.$, $\left.\rightarrow_{152, \alpha, \beta}, \rightarrow_{153, \varepsilon, \eta}\right)$ contain parameters and by this reason the method used here is not applicable to them. Their properties will be discussed in a next research.

Here, we give the 17 axioms of the intuitionistic logic (see, e.g. [6]) If $A, B$ and $C$ are arbitrary propositional forms, then:
(a) $A \rightarrow A$,
(b) $A \rightarrow(B \rightarrow A)$,
(c) $A \rightarrow(B \rightarrow(A \& B))$,
(d) $(A \rightarrow(B \rightarrow C)) \rightarrow(B \rightarrow(A \rightarrow C))$,
(e) $(A \rightarrow(B \rightarrow C)) \rightarrow((A \rightarrow B) \rightarrow(A \rightarrow C))$,
(f) $A \rightarrow \neg \neg A$,
(g) $\neg(A \& \neg A)$,
(h) $(\neg A \vee B) \rightarrow(A \rightarrow B)$,
(i) $\neg(A \vee B) \rightarrow(\neg A \& \neg B)$,
(j) $(\neg A \& \neg B) \rightarrow \neg(A \vee B)$,
(k) $(\neg A \vee \neg B) \rightarrow \neg(A \& B)$
(l) $(A \rightarrow B) \rightarrow(\neg B \rightarrow \neg A)$,
(m) $(A \rightarrow \neg B) \rightarrow(B \rightarrow \neg A)$,
(n) $\neg \neg \neg A \rightarrow \neg A$,
(o) $\neg A \rightarrow \neg \neg \neg A$,
(p) $\neg \neg(A \rightarrow B) \rightarrow(A \rightarrow \neg \neg B)$,
(q) $(C \rightarrow A) \rightarrow((C \rightarrow(A \rightarrow B)) \rightarrow(C \rightarrow B))$.

Now, we formulate the following

Theorem. The intuitionistic fuzzy implications (from Table 1) and negations (from Table 2) related to them (see Table 3) that satisfy the axioms of intuitionistic logic as (classical) tautologies, are marked in Table 4 by "•" and these that satisfy the same axioms (only) as IFTs - by "०".

Table 4

\begin{tabular}{|c|c|c|c|c|c|c|c|c|c|c|c|c|c|c|c|c|c|}
\hline & $\mathrm{a}$ & & $\mathrm{c}$ & $\mathrm{d}$ & $\mathrm{e}$ & f & & $\mathrm{h}$ & i & & & & $\mathrm{m}$ & $\mathrm{n}$ & $\mathrm{O}$ & $\mathrm{p}$ & q \\
\hline$\rightarrow_{1}$ & • & o & o & o & $\bullet$ & $\bullet$ & $\bullet$ & o & $\bullet$ & $\bullet$ & $\bullet$ & $\bullet$ & $\bullet$ & $\bullet$ & $\bullet$ & $\bullet$ & $\bullet$ \\
\hline$\rightarrow_{2}$ & $\bullet$ & & & & $\bullet$ & $\bullet$ & $\bullet$ & & $\bullet$ & 0 & $\bullet$ & $\bullet$ & $\bullet$ & $\bullet$ & $\bullet$ & $\bullet$ & $\bullet$ \\
\hline$\rightarrow_{3}$ & 0 & O & o & o & 0 & o & O & 0 & 0 & 0 & 0 & O & o & O & O & 0 & O \\
\hline$\rightarrow_{4}$ & 0 & 0 & o & o & 0 & o & o & 0 & 0 & 0 & o & ○ & o & O & O & 0 & O \\
\hline$\rightarrow_{5}$ & 0 & 0 & o & o & 0 & o & O & 0 & 0 & 0 & 0 & O & o & O & 0 & 0 & O \\
\hline$\rightarrow_{6}$ & 0 & 0 & o & o & & o & o & 0 & o & o & o & ○ & o & O & O & 0 & \\
\hline$\rightarrow_{7}$ & 0 & o & & & & o & O & & O & 0 & 0 & O & O & O & 0 & 0 & \\
\hline$\rightarrow_{8}$ & $\bullet$ & & & & & $\bullet$ & $\bullet$ & & $\bullet$ & $\bullet$ & $\bullet$ & $\bullet$ & $\bullet$ & $\bullet$ & $\bullet$ & $\bullet$ & \\
\hline$\rightarrow_{9}$ & 0 & o & & o & o & o & O & o & 0 & 0 & 0 & O & O & O & 0 & 0 & O \\
\hline$\rightarrow_{10}$ & & & & & & & 0 & & & & & & & & & & \\
\hline$\rightarrow_{11}$ & $\bullet$ & $\bullet$ & $\bullet$ & $\bullet$ & $\bullet$ & $\bullet$ & $\bullet$ & $\bullet$ & $\bullet$ & $\bullet$ & $\bullet$ & $\bullet$ & $\bullet$ & $\bullet$ & $\bullet$ & $\bullet$ & $\bullet$ \\
\hline$\rightarrow_{12}$ & & & & 0 & 0 & 0 & 0 & & 0 & O & 0 & 0 & O & 0 & 0 & 0 & 0 \\
\hline$\rightarrow_{13}$ & 0 & 0 & O & 0 & 0 & o & 0 & 0 & 0 & 0 & 0 & ○ & 0 & 0 & 0 & 0 & O \\
\hline$\rightarrow_{14}$ & $\bullet$ & $\bullet$ & $\bullet$ & $\bullet$ & $\bullet$ & $\bullet$ & $\bullet$ & $\bullet$ & $\bullet$ & $\bullet$ & $\bullet$ & - & $\bullet$ & $\bullet$ & $\bullet$ & $\bullet$ & $\bullet$ \\
\hline$\rightarrow_{15}$ & $\bullet$ & & & & & $\bullet$ & $\bullet$ & & $\bullet$ & $\bullet$ & $\bullet$ & $\bullet$ & $\bullet$ & $\bullet$ & $\bullet$ & $\bullet$ & \\
\hline$\rightarrow_{16}$ & & & & & & $\bullet$ & • & & $\bullet$ & $\bullet$ & $\bullet$ & $\bullet$ & $\bullet$ & $\bullet$ & $\bullet$ & $\bullet$ & \\
\hline$\rightarrow_{17}$ & 0 & 0 & 0 & 0 & 0 & 0 & 0 & 0 & 0 & 0 & 0 & 0 & 0 & 0 & 0 & 0 & 0 \\
\hline$\rightarrow_{18}$ & 0 & 0 & 0 & 0 & 0 & 0 & 0 & 0 & 0 & 0 & 0 & ○ & 0 & 0 & 0 & 0 & 0 \\
\hline$\rightarrow_{19}$ & & & & & & $\bullet$ & $\bullet$ & & $\bullet$ & $\theta$ & $\bullet$ & $\bullet$ & $\bullet$ & $\bullet$ & $\bullet$ & $\bullet$ & \\
\hline$\rightarrow_{20}$ & $\bullet$ & $\bullet$ & $\bullet$ & $\bullet$ & $\bullet$ & $\bullet$ & $\bullet$ & $\bullet$ & $\bullet$ & $\bullet$ & $\bullet$ & $\bullet$ & - & $\bullet$ & $\bullet$ & $\bullet$ & $\bullet$ \\
\hline
\end{tabular}

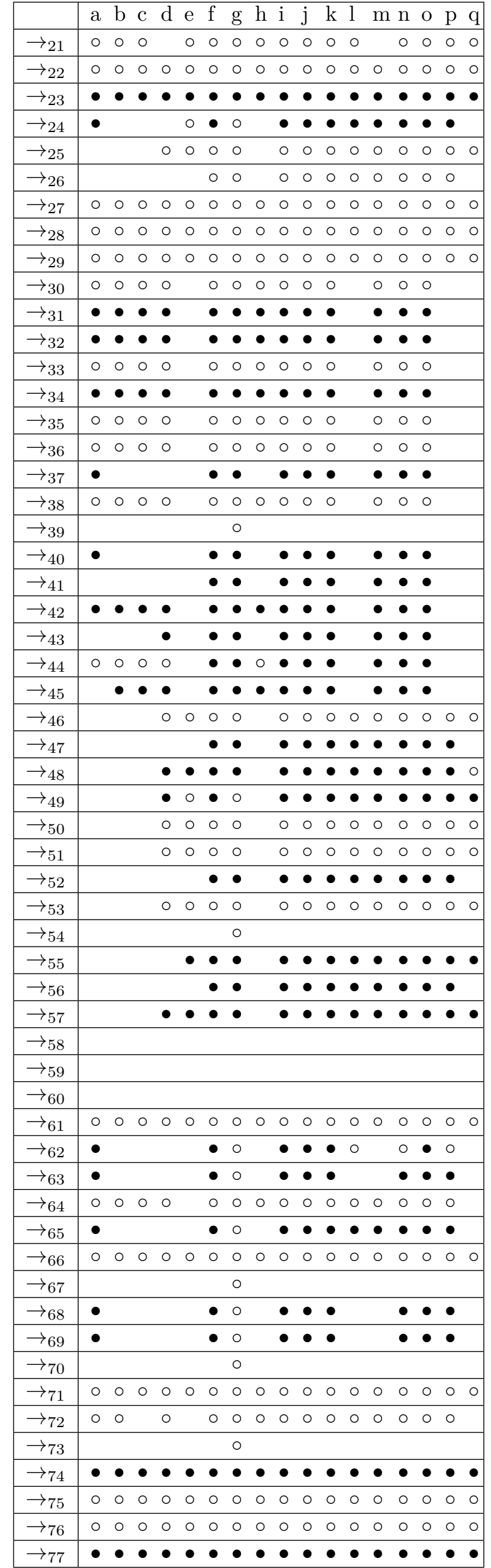




\begin{tabular}{|c|c|c|c|c|c|c|c|c|c|c|c|c|c|c|c|c|c|}
\hline & a & $\mathrm{b}$ & & $\mathrm{d}$ & $\mathrm{e}$ & $\mathrm{f}$ & 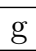 & $\mathrm{h}$ & $\mathrm{i}$ & $\mathrm{j}$ & $\mathrm{k}$ & 1 & $\mathrm{n}$ & $\mathrm{n}$ & $\mathrm{O}$ & $\mathrm{p}$ & $\mathrm{q}$ \\
\hline$\rightarrow_{78}$ & & & & & & & $\Omega$ & & & & & & & & & & \\
\hline$\rightarrow_{79}$ & 0 & 0 & 0 & 0 & 0 & 0 & 0 & 0 & 0 & 0 & 0 & 0 & 0 & 0 & 0 & 0 & 0 \\
\hline$\rightarrow_{80}$ & O & 0 & & 0 & & 0 & 0 & 0 & 0 & 0 & 0 & 0 & 0 & 0 & 0 & 0 & \\
\hline$\rightarrow_{81}$ & O & 0 & 0 & 0 & 0 & 0 & 0 & 0 & 0 & O & 0 & 0 & 0 & 0 & 0 & 0 & 0 \\
\hline$\rightarrow_{82}$ & O & 0 & 0 & 0 & & 0 & 0 & 0 & 0 & 0 & 0 & & 0 & 0 & 0 & & \\
\hline$\rightarrow_{83}$ & $\bullet$ & & & & & $\bullet$ & $\bullet$ & & - & $\bullet$ & $\bullet$ & & $\bullet$ & $\bullet$ & $\bullet$ & & \\
\hline$\rightarrow_{84}$ & $\bullet$ & & & & & $\bullet$ & 0 & & $\bullet$ & $\bullet$ & $\bullet$ & & & $\bullet$ & $\bullet$ & & \\
\hline$\rightarrow_{85}$ & O & 0 & 0 & 0 & & 0 & 0 & 0 & O & 0 & 0 & & 0 & 0 & 0 & & \\
\hline$\rightarrow_{86}$ & & & & & & & O & & & & & & & & & & \\
\hline$\rightarrow_{87}$ & & & & & & & 0 & & & & & & & & & & \\
\hline$\rightarrow_{88}$ & $\bullet$ & $\bullet$ & $\bullet$ & $\bullet$ & & $\bullet$ & $\bullet$ & $\bullet$ & • & $\bullet$ & $\bullet$ & & $\bullet$ & $\bullet$ & $\bullet$ & & \\
\hline$\rightarrow_{89}$ & 0 & 0 & & 0 & & 0 & 0 & 0 & 0 & 0 & 0 & & 0 & 0 & 0 & & \\
\hline$\rightarrow 90$ & 0 & 0 & 0 & $\bullet$ & & $\bullet$ & $\bullet$ & 0 & $\bullet$ & $\bullet$ & $\bullet$ & & $\bullet$ & $\bullet$ & $\bullet$ & & \\
\hline$\rightarrow_{91}$ & & & & 0 & 0 & 0 & 0 & & o & 0 & 0 & 0 & 0 & 0 & 0 & 0 & \\
\hline$\rightarrow_{92}$ & & & & & & & & & & & & & & & & & \\
\hline$\rightarrow_{93}$ & & & & & & $\bullet$ & 0 & & $\bullet$ & $\bullet$ & $\bullet$ & & & $\bullet$ & $\bullet$ & $\bullet$ & \\
\hline$\rightarrow 94$ & & & & 0 & 0 & 0 & 0 & & 0 & 0 & 0 & 0 & 0 & 0 & 0 & 0 & \\
\hline$\rightarrow_{95}$ & & & & & & & 0 & & & & & & & & & & \\
\hline$\rightarrow_{96}$ & & & & & & & 0 & & & & & & & & & & \\
\hline$\rightarrow 97$ & & & & $\bullet$ & $\bullet$ & $\bullet$ & $\bullet$ & & $\bullet$ & $\bullet$ & $\bullet$ & $\bullet$ & $\bullet$ & $\bullet$ & $\bullet$ & $\bullet$ & $\bullet$ \\
\hline$\rightarrow_{98}$ & & & & & & 0 & 0 & & 0 & O & 0 & & & 0 & $\bullet$ & & \\
\hline$\rightarrow_{99}$ & & & & & & 0 & 0 & & & & & & & & 0 & & \\
\hline$\rightarrow_{100}$ & O & 0 & 0 & 0 & 0 & 0 & 0 & 0 & 0 & 0 & 0 & 0 & 0 & 0 & 0 & 0 & \\
\hline$\rightarrow_{101}$ & O & 0 & 0 & 0 & 0 & 0 & 0 & 0 & o & O & 0 & 0 & 0 & 0 & 0 & 0 & O \\
\hline$\rightarrow_{102}$ & 0 & 0 & 0 & 0 & 0 & 0 & 0 & 0 & 0 & 0 & 0 & 0 & 0 & 0 & 0 & 0 & 0 \\
\hline$\rightarrow_{103}$ & 0 & 0 & 0 & 0 & & 0 & 0 & 0 & O & O & 0 & & 0 & 0 & 0 & & \\
\hline$\rightarrow_{104}$ & 0 & 0 & 0 & 0 & & 0 & 0 & 0 & o & O & 0 & 0 & 0 & 0 & 0 & 0 & \\
\hline$\rightarrow_{105}$ & 0 & 0 & 0 & 0 & & 0 & 0 & 0 & 0 & 0 & 0 & 0 & 0 & 0 & 0 & 0 & \\
\hline$\rightarrow_{106}$ & & & & 0 & 0 & 0 & 0 & & & & 0 & & 0 & 0 & 0 & & \\
\hline$\rightarrow_{107}$ & & & & 0 & & 0 & 0 & 0 & o & o & 0 & & 0 & 0 & 0 & 0 & \\
\hline$\rightarrow_{108}$ & & & & & & 0 & 0 & & & & & & & & 0 & & \\
\hline$\rightarrow_{109}$ & 0 & 0 & 0 & 0 & 0 & 0 & 0 & 0 & 0 & 0 & 0 & 0 & 0 & 0 & 0 & 0 & \\
\hline$\rightarrow_{110}$ & 0 & 0 & 0 & 0 & 0 & 0 & 0 & 0 & o & o & 0 & 0 & 0 & 0 & 0 & 0 & \\
\hline$\rightarrow_{111}$ & O & 0 & 0 & 0 & 0 & 0 & 0 & 0 & 0 & o & 0 & 0 & 0 & 0 & 0 & $0 c$ & \\
\hline$\rightarrow_{112}$ & 0 & 0 & 0 & 0 & 0 & 0 & 0 & 0 & o & o & 0 & 0 & 0 & 0 & 0 & 00 & o \\
\hline$\rightarrow_{113}$ & O & 0 & 0 & o & o & 0 & 0 & 0 & o & o & O & 0 & 0 & 0 & 0 & $0 c$ & O \\
\hline$\rightarrow_{114}$ & o & 0 & 0 & 0 & & 0 & 0 & 0 & 0 & 0 & 0 & & 0 & 0 & 0 & & \\
\hline$\rightarrow_{115}$ & 0 & 0 & 0 & 0 & & 0 & 0 & 0 & 0 & o & 0 & & 0 & 0 & 0 & & \\
\hline$\rightarrow_{116}$ & O & 0 & 0 & o & & 0 & 0 & 0 & O & o & 0 & & 0 & 0 & 0 & & \\
\hline$\rightarrow_{117}$ & O & 0 & 0 & 0 & & 0 & 0 & 0 & 0 & 0 & 0 & & 0 & 0 & 0 & & \\
\hline$\rightarrow_{118}$ & 0 & 0 & 0 & 0 & 0 & 0 & 0 & 0 & 0 & 0 & 0 & 0 & 0 & 0 & 0 & $0 \mathrm{c}$ & 0 \\
\hline$\rightarrow_{119}$ & & & & & & 0 & 0 & & 0 & 0 & 0 & & & 0 & 0 & 0 & \\
\hline$\rightarrow_{120}$ & & & & 0 & & 0 & 0 & & 0 & o & 0 & 0 & 0 & 0 & 0 & 0 & \\
\hline$\rightarrow_{121}$ & & & & 0 & 0 & 0 & 0 & & 0 & 0 & 0 & 0 & 0 & 0 & 0 & $0 \mathrm{c}$ & \\
\hline$\rightarrow_{122}$ & & & & 0 & & 0 & 0 & & O & o & 0 & 0 & 0 & 0 & 0 & 0 & \\
\hline$\rightarrow_{123}$ & & & & & & 0 & 0 & & o & O & 0 & & & 0 & 0 & & \\
\hline$\rightarrow_{124}$ & 0 & 0 & 0 & 0 & 0 & 0 & 0 & 0 & 0 & 0 & 0 & 0 & 0 & 0 & 0 & $0 \mathrm{c}$ & \\
\hline$\rightarrow_{125}$ & O & 0 & 0 & o & 0 & 0 & 0 & 0 & o & o & 0 & 0 & 0 & 0 & 0 & 0 & \\
\hline$\rightarrow_{126}$ & 0 & 0 & 0 & O & 0 & 0 & 0 & 0 & o & O & 0 & 0 & 0 & 0 & 0 & $0 c$ & o \\
\hline$\rightarrow_{127}$ & 0 & 0 & 0 & 0 & 0 & 0 & 0 & 0 & 0 & 0 & 0 & 0 & 0 & 0 & 0 & 0 & \\
\hline$\rightarrow_{128}$ & 0 & 0 & 0 & O & 0 & 0 & 0 & 0 & o & O & 0 & 0 & 0 & 0 & 0 & $0 \mathrm{c}$ & \\
\hline$\rightarrow_{129}$ & o & 0 & 0 & 0 & & 0 & 0 & 0 & o & o & 0 & & 0 & 0 & 0 & & \\
\hline$\rightarrow_{130}$ & O & 0 & 0 & O & & 0 & 0 & 0 & O & O & 0 & & 0 & 0 & 0 & & \\
\hline$\rightarrow_{131}$ & 0 & 0 & 0 & 0 & & 0 & 0 & 0 & 0 & 0 & 0 & & 0 & 0 & 0 & & \\
\hline$\rightarrow_{132}$ & O & 0 & o & O & & o & 0 & 0 & O & o & o & & O & 0 & 0 & & \\
\hline$\rightarrow_{133}$ & O & 0 & 0 & O & & o & 0 & 0 & O & O & 0 & O & 0 & 0 & 0 & 0 & \\
\hline$\rightarrow_{134}$ & & & & 0 & 0 & 0 & 0 & & 0 & O & 0 & 0 & 0 & 0 & 0 & $0 \mathrm{c}$ & \\
\hline$\rightarrow_{135}$ & & & & O & 0 & 0 & & & o & o & o & 0 & ○ & ○ & 0 & $0 c$ & \\
\hline
\end{tabular}

\begin{tabular}{|c|c|c|c|c|c|c|c|c|c|c|c|c|c|c|}
\hline & a $\quad b \quad c \quad d$ & $\mathrm{e}$ & $\mathrm{f}$ & $\mathrm{g} h$ & $\mathrm{i}$ & $\mathrm{j}$ & $\mathrm{k}$ & 1 & & $\mathrm{~m}$ & $\mathrm{n}$ & $\mathrm{O}$ & D & $\mathrm{q}$ \\
\hline$\rightarrow_{136}$ & O & 0 & 0 & 0 & 0 & 0 & 0 & 0 & & 0 & 0 & O & O & 0 \\
\hline$\rightarrow_{137}$ & 0 & 0 & 0 & 0 & 0 & 0 & 0 & $c$ & & 0 & 0 & 0 & O & 0 \\
\hline$\rightarrow_{138}$ & & & & 0 & & & & & & & & & & \\
\hline$\rightarrow_{139}$ & 0 & & & & 0 & 0 & 0 & & & & & & & \\
\hline$\rightarrow_{140}$ & & & & & & & & & & & & & & \\
\hline$\rightarrow_{141}$ & 0 & & & & 0 & 0 & 0 & & & & 0 & & & \\
\hline$\rightarrow_{142}$ & & & & & & & & & & & & & & \\
\hline$\rightarrow_{143}$ & & & & & & & . & & & & & & & \\
\hline$\rightarrow_{145}$ & & & & & & & & & & & & & & \\
\hline$\rightarrow_{146}$ & 0 & & & & 0 & 0 & 0 & & & & 0 & & & \\
\hline$\rightarrow_{147}$ & 0 & & & & 0 & 0 & 0 & & & & 0 & & & \\
\hline$\rightarrow_{148}$ & 0 & & & & 0 & 0 & 0 & & & & 0 & & & \\
\hline$\rightarrow_{149}$ & & & & & 0 & 0 & O & & & & 0 & & & \\
\hline
\end{tabular}

The check of the validity of the assertion in Theorem 1 was made by the software application IFSTool $[5,9]$, developed as a tool for automatic check of the properties of intuitionistic fuzzy implications and negations. The software has an option - to either check for intuitionistic fuzzy tautologies or only for fuzzy tautologies. For the needs of the present paper, each axiom was tested with all implications and their corresponding negations, and manual backup checks of some of the properties were made, as well.

On the other hand, each one of the above mentioned checks can be realized directly. For example, for implication $\rightarrow_{16}$, Axiom (f) is checked as follows. It has the form (see Table 1):

$$
\langle a, b\rangle \rightarrow_{16}\langle c, d\rangle=\langle\max (\overline{\operatorname{sg}}(a), c), \min (\operatorname{sg}(a), d)\rangle
$$

and it generates (see Table 3 ) the negation $\neg_{2}$ with the form (see Table 2):

$$
\neg_{2}\langle a, b\rangle=\langle\overline{\operatorname{sg}}(a), \operatorname{sg}(a)\rangle .
$$

Therefore, we construct the expression

$$
\begin{gathered}
A \rightarrow \neg \neg A=\langle a, b\rangle \rightarrow_{16} \neg_{2} \neg_{2}\langle a, b\rangle \\
=\langle a, b\rangle \rightarrow_{16} \neg_{2}\langle\overline{\operatorname{sg}}(a), \operatorname{sg}(a)\rangle \\
=\langle a, b\rangle \rightarrow_{16}\langle\overline{\operatorname{sg}}(\overline{\operatorname{sg}}(a)), \operatorname{sg}(\overline{\operatorname{sg}}(a))\rangle \\
=\langle a, b\rangle \rightarrow_{16}\langle\operatorname{sg}(a), \overline{\operatorname{sg}}(a)\rangle \\
=\langle\max (\overline{\operatorname{sg}}(a), \operatorname{sg}(a)), \min (\operatorname{sg}(a), \overline{\operatorname{sg}}(a))\rangle \\
=\langle 1,0\rangle .
\end{gathered}
$$

All the rest checks are similar, but, of course, in some cases, they are essentially longer and difficult. For the case of intuitionistic fuzzy sets, the intuitionistic logic axioms were checked for a part of the above implications and negations in [1].

The Theorem gives us information which implications satisfy all, no one or part of the axions of intuitionistic logic. Therefore, we can call the implications that satisfy all (part of; or no one of) axions of the intuitionistic logic as tautologies or as IFTs, respectively, tautology- (or IFT-) good, partly good or bad implications from intuitionistic logic point of view. 


\section{Conclusion: Open problems}

We finish with three interesting open problems.

Open problem 1. In classical logic, the operation "disjunction" is obtained by the formula $x \vee y=$ $\neg x \rightarrow y$. Which will be the form of the new disjunctions, that can be generated by the above implications?

Open problem 2. The same question is interesting for the new possible operations "conjunction".

In both cases, probably, from some (different) implication coinciding conjunctions or disjunctions will be generated. So, one of them must be eliminated in the obtained list of new operations.

Open problem 3. Will the new disjunctions generate new implications using the classical logical formula $x \rightarrow y=\neg x \vee y$ ?

Open problem 4. Which applications in practice can find the separate and more essentially, the tautology- (or IFT-) good implications from intuitionistic logic point of view?

In a next research, other properties of the implications will be studied. The present research will be an object of future research from point of view of the InterCriteria Analysis (see [4]).

\section{Acknowledgements}

The authors are thankful for the support provided by the Bulgarian National Science Fund under Grant Ref. No. DFNI-I-02-5 "InterCriteria Analysis: A New Approach to Decision Making".

\section{References}

[1] K. Atanassov. On Intuitionistic Fuzzy Sets Theory, Berlin: Springer, 2012.

[2] K. Atanassov. On Intuitionistic Fuzzy Logics: Results and Problems. In: Modern Approaches in Fuzzy Sets, Intuitionistic Fuzzy Sets, Generalized Nets and Related Topics, Volume 1: Foundations. (Atanassov, K., M. Baczynski, J. Drewniak, J. Kacprzyk, M. Krawczak, E. Szmidt, M. Wygralak, S. Zadrozny, eds.), SRIPAS, Warsaw, pages 23-49, 2014.

[3] K. Atanassov. On a new intuitionistic fuzzy implication. (submitted for participation in IFSAEUSFLAT 2015)

[4] Atanassov K., D. Mavrov, V. Atanassova. Intercriteria Decision Making: A New Approach for Multicriteria Decision Making, Based on Index Matrices and Intuitionistic Fuzzy Sets. Issues in Intuitionistic Fuzzy Sets and Generalized Nets, Vol. 11, 2014, 1-8.

[5] D. Dimitrov. IFSTool - Software for intuitionistic fuzzy sets. Issues in Intuitionistic Fuzzy Sets and Generalized Nets, 9: 61-69, 2011.
[6] H. Rasiova, and R. Sikorski. The Mathematics of Metamathematics, Warszawa, Pol. Acad. of Sci., 1963.

[7] Implications over intuitionistic fuzzy sets. Ifigenia.net. Available at: http://www.ifigenia.org/ wiki/IF_implications

[8] Negations over intuitionistic fuzzy sets. Ifigenia.net. Available at: http://www.ifigenia.org/ wiki/IF_negations

[9] IFSTool. Ifigenia.net. Available at: http://www. ifigenia.org/wiki/IFSTool 